\title{
THE ANTI-CRISIS ROLE OF THE STATE (BASED ON THE EXPERIENCE OF THE GLOBAL FINANCIAL AND THE CORONOMIC CRISIS)
}

\author{
ELGUJA MEKVABISHVILI \\ Doctor of Economic Sciences, Professor, \\ Academician of Georgia Economic Sciences Academy \\ Ivane Javakhishvili Tbilisi State University, Georgia \\ elguja.mekvabishvili@tsu.ge
}

Abstract. A system that is a mixed economy has established in modern post-industrial countries and the participation of the state in it is very important. The active role of the state is especially evident in extreme situations, as evidenced by the experience of the most acute of the 21st century - the global financial crisis and the coronary crisis caused by the Covid-19 pandemic. Against the background of the implemented and ongoing changes in the economies of world and nation states in economic theory today we see the revival of the Keynesian doctrine. This doctrine withdrew the United States of America, as well as other countries, out of the worst crisis of the 20th century, out of the so-called "The Great Depression" and it has been a major factor in the unprecedented economic success of these countries during four decades.

A comparative analysis of the global financial and economic crises shows that the state not only plays a leading role in the process of rescuing economies from the crisis, but also its participation in post-crisis development, which implies the so-called Performing the function of «bridge management».

Based on the experience of the coronary crisis, in order to ensure the stability and development of the Georgian economy, we consider it necessary:

- Based on finding and using local resources, the state should implement economic policies aimed at accelerating the development of the real sector of the economy, diversifying the internal food market, reducing the negative foreign trade balance of agri-food products. This does not mean prioritizing import-substituting production, but focusing on the development of local production of products whose resources currently exist in our country;

- The state must create strategic reserves of food, medical supplies and basic necessities, which are vital in a rapidly changing global environment full of uncertainty and risk;

- Attitudes towards the tourism sector need to be changed substantially. After the end of the pandemic, the world will return to normal life and the number of tourists entering Georgia will increase again. However, given the lessons of the pandemic, we must bear in mind that it is necessary to: a) move to an intensive model of tourism; B) Organic inclusion of tourism in the local value chain; C) Pay more attention to the development of local tourism.

KEYWORDS: ANTI-CRISIS ROLE OF THE STATE, CORONOMIC CRISES, “OMNIPOTENT STATE”.

For citation: Mekvabishvili, E., (2020). The Anti-Crisis Role of the State (Based on the Experience of the Global Financial and the Coronomic Crisis. Globalization and Business, 10. 35-41. (In Georgian) https://doi.org/10.35945/gb.2020.10.003 


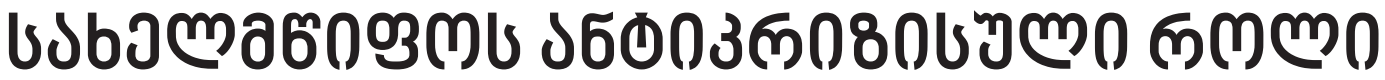

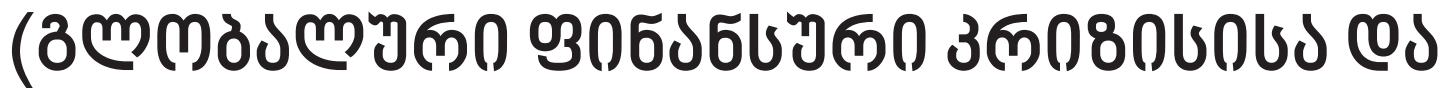

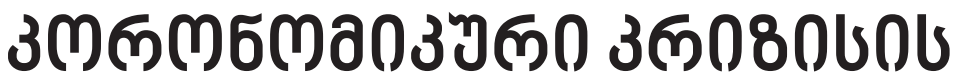

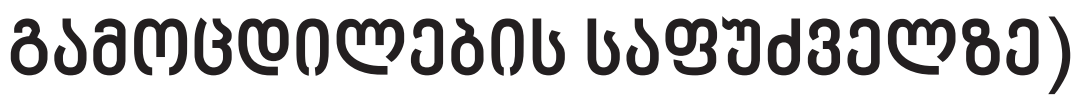

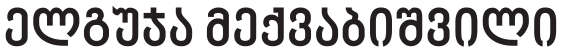

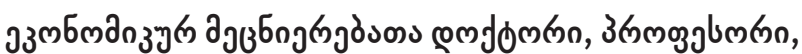

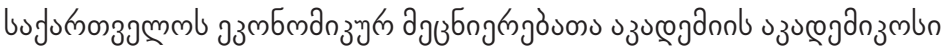

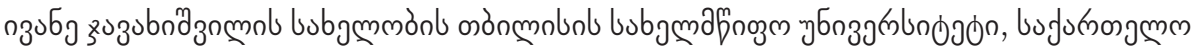

elguja.mekvabishvili@tsu.ge

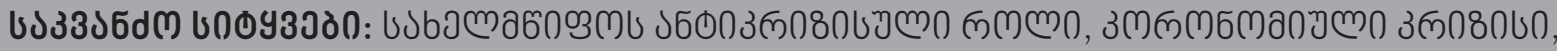

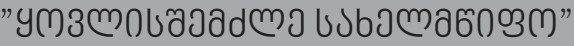

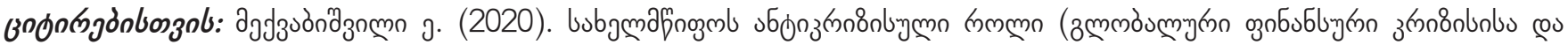

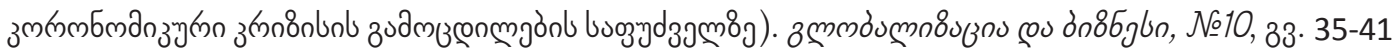

https://doi.org/10.35945/gb.2020.10.003

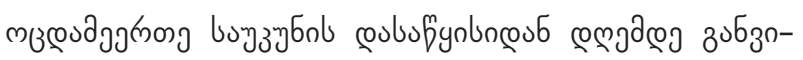

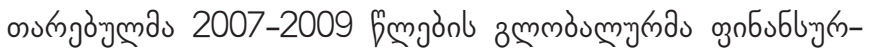
ззмбмanзचु

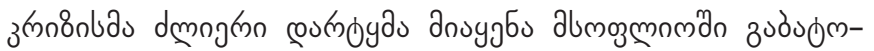

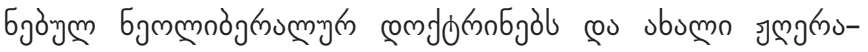

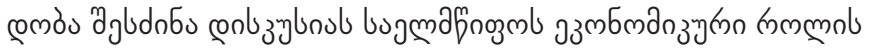

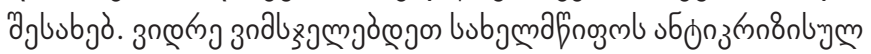

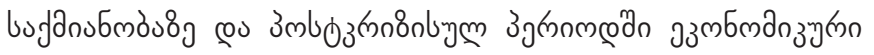

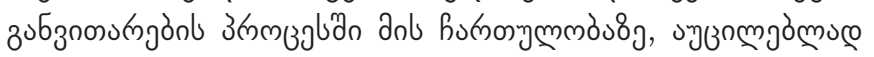

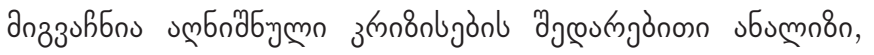

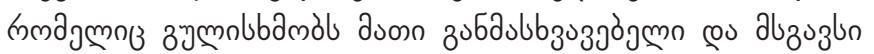

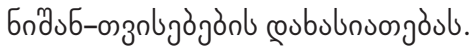

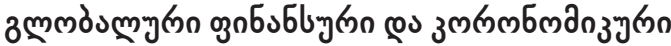

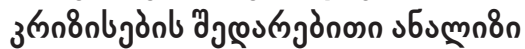

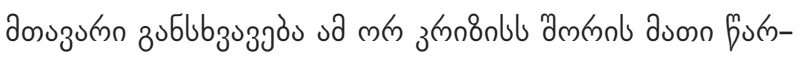

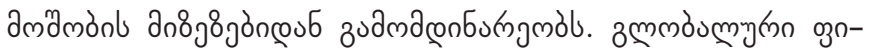

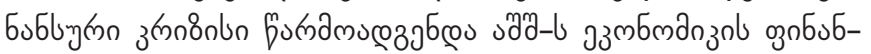

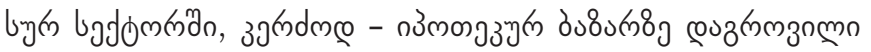

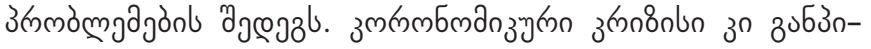

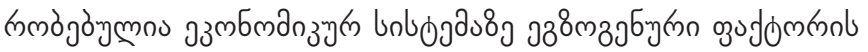

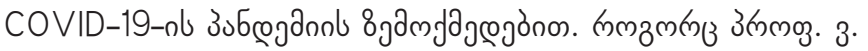

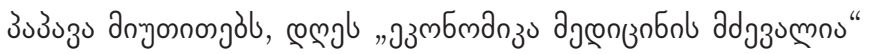
(https://for.ge/view/182671/koronomika-da-ekonomika-

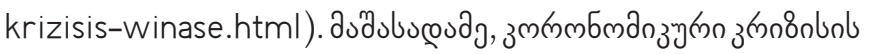

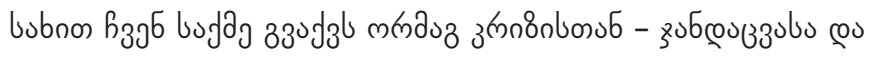
ззмбмдаз зада.

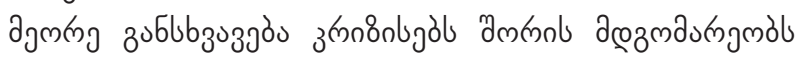

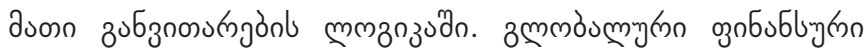

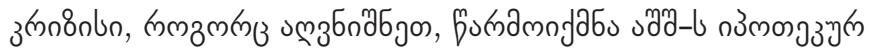

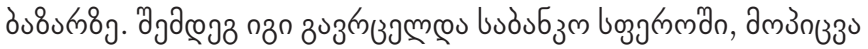

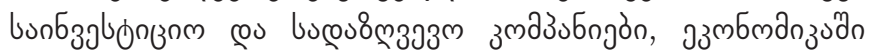

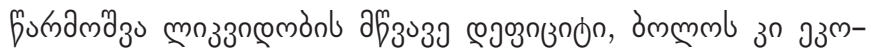

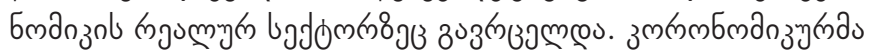

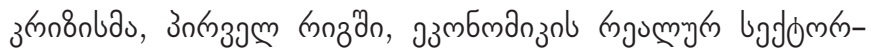

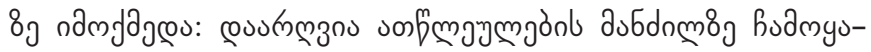

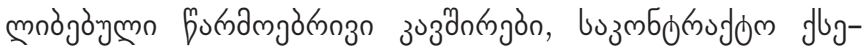

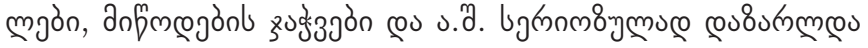

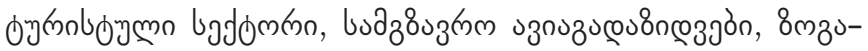

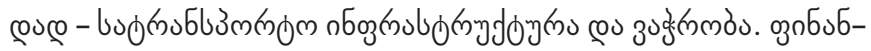

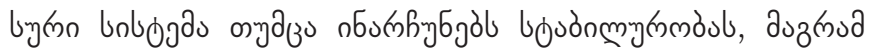

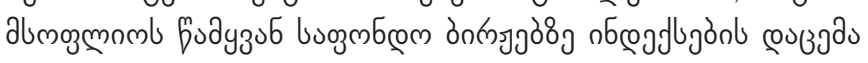

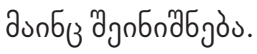

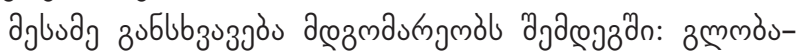

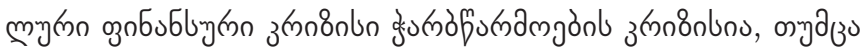

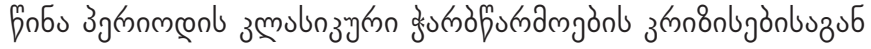

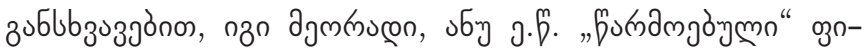

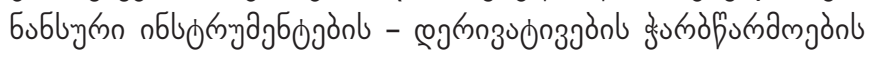

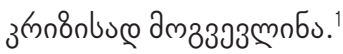

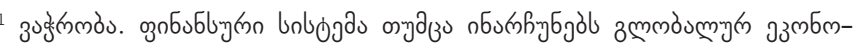

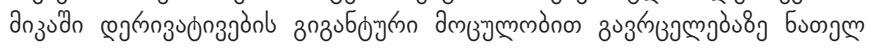




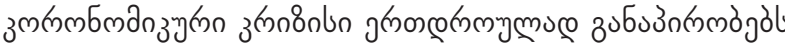

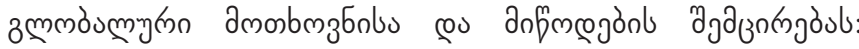

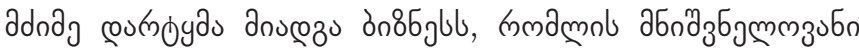

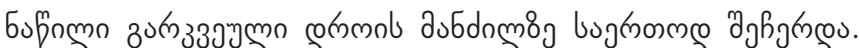

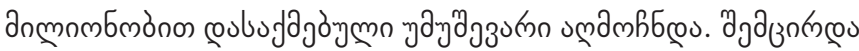

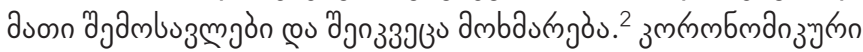

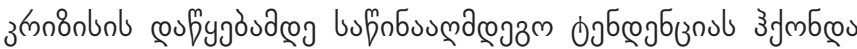

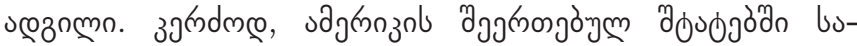

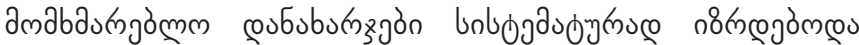

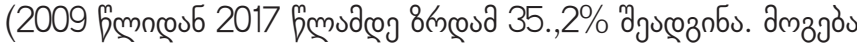

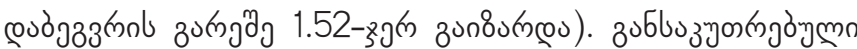

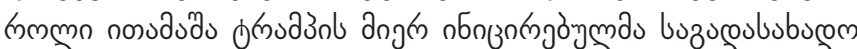

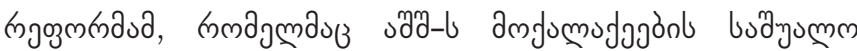

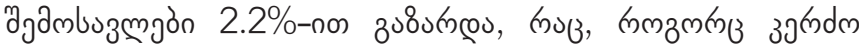
उntrossonzol, n

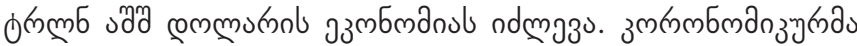
з ऊnon

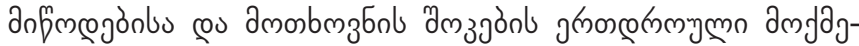

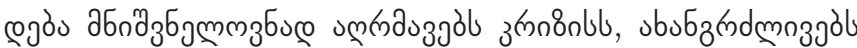

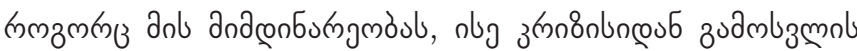

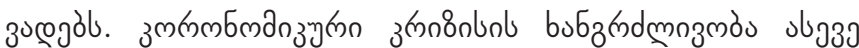

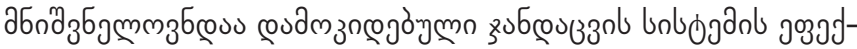
on

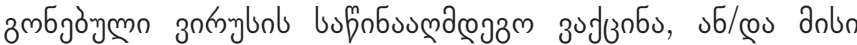

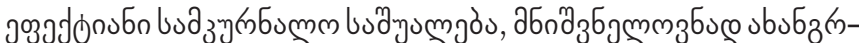

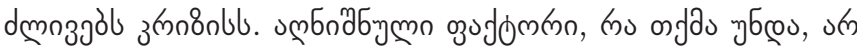

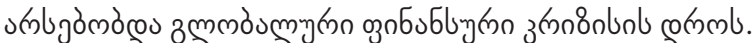

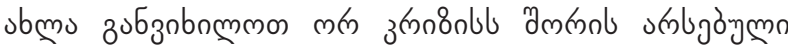

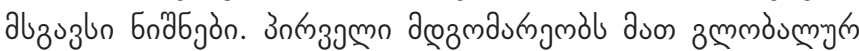

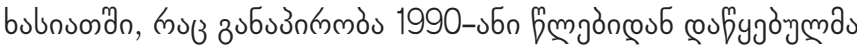

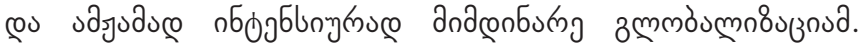

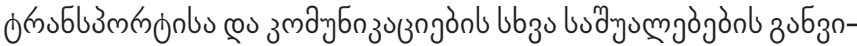

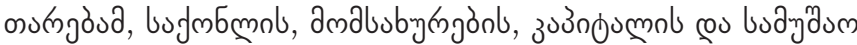

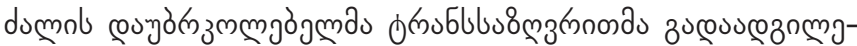

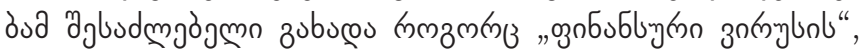

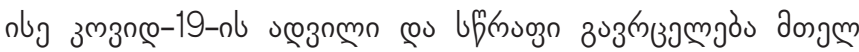

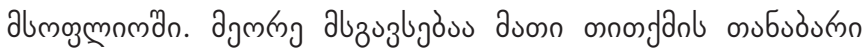
ипмп

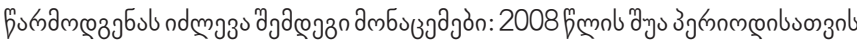

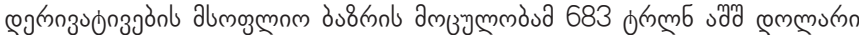
zou@z

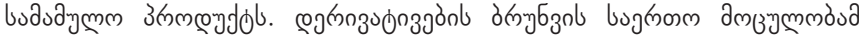

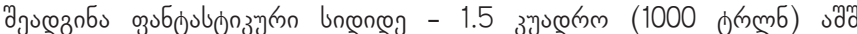

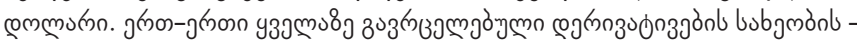

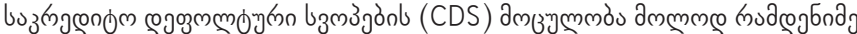

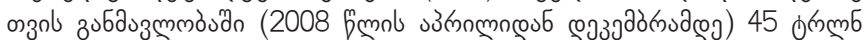

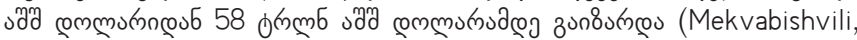

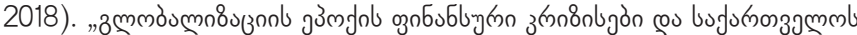
эзмбмдаз зи". 33. 100-101.)

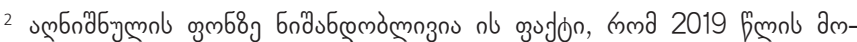

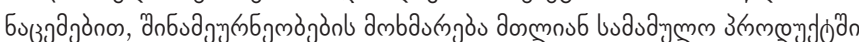

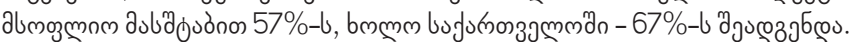

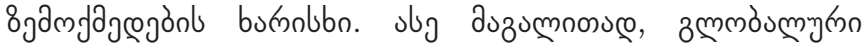

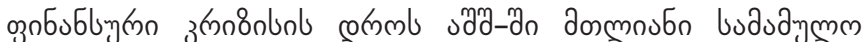

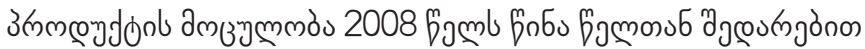

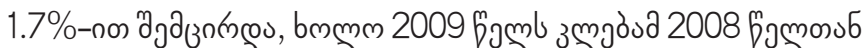

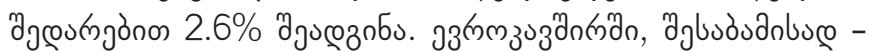

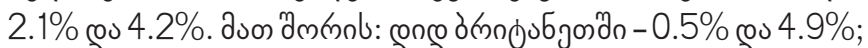
зु

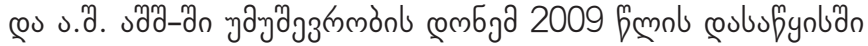

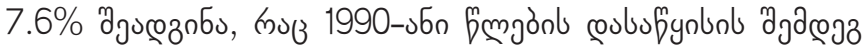

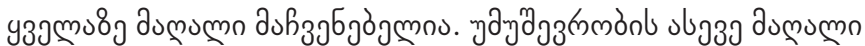

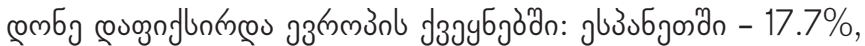

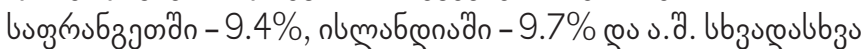

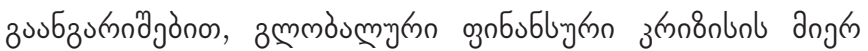

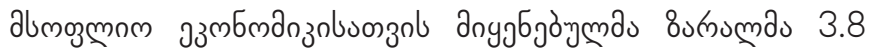

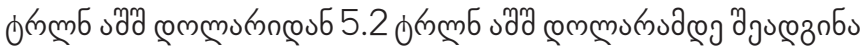
(Monitoring the World Crisis, 2010: 6-7).

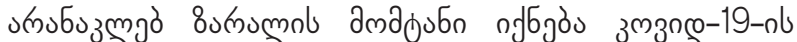

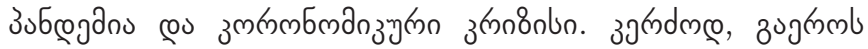

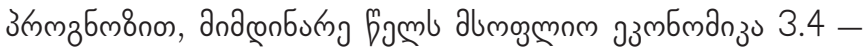

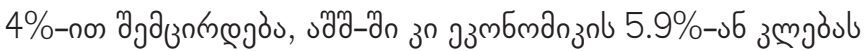

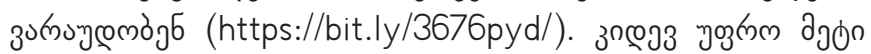

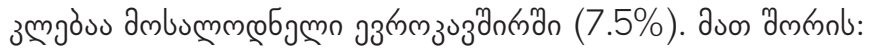

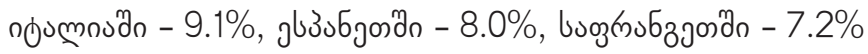

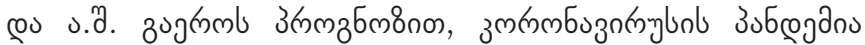

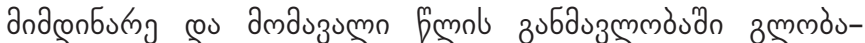

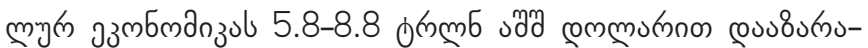

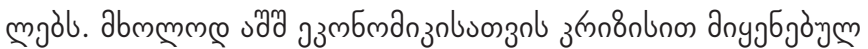
ambummmoб

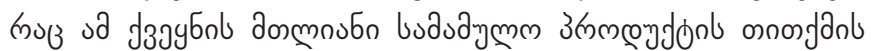
10\%-nu (https://bit.ly/2TcSmoqu). abmogmnn 3ugf(mmònl

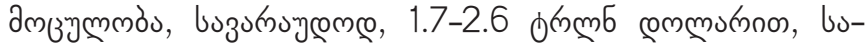

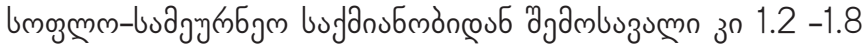

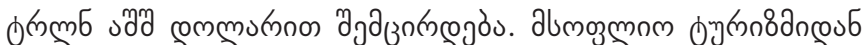

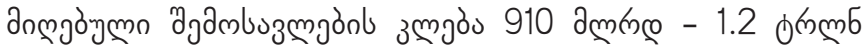

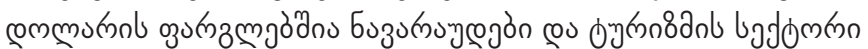

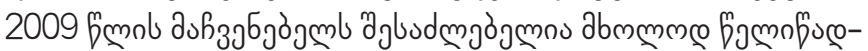

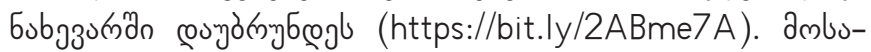

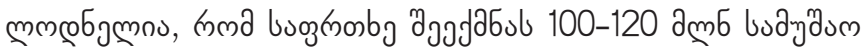
ucangmb.

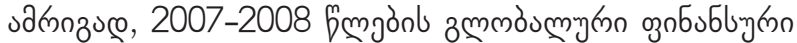

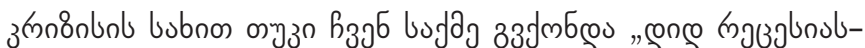

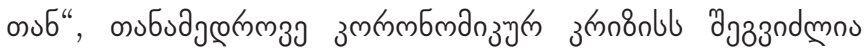

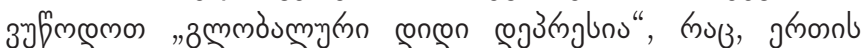

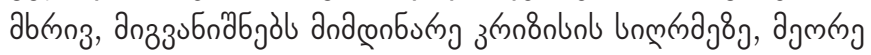

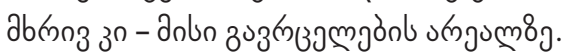

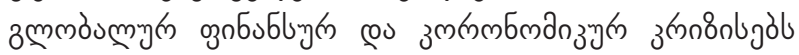

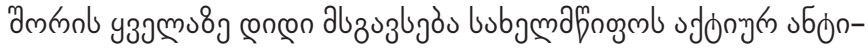

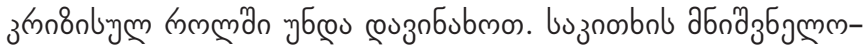

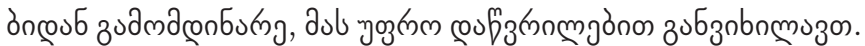




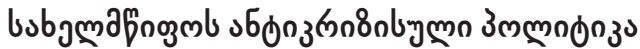

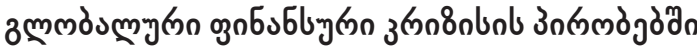

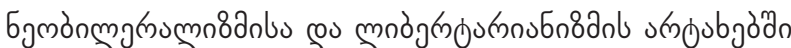

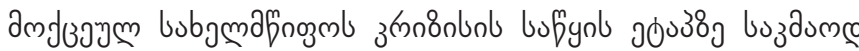

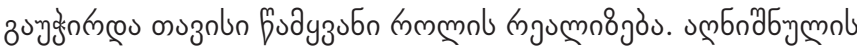

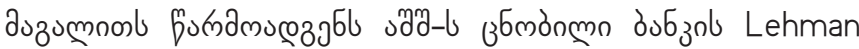

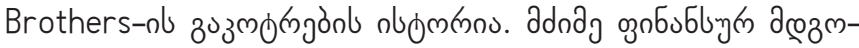

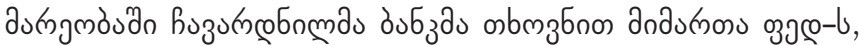

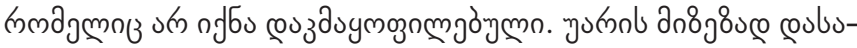

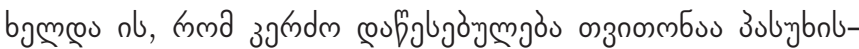

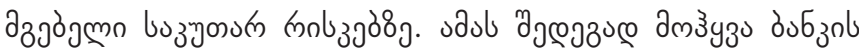

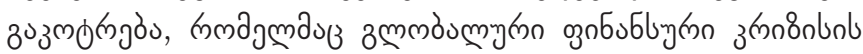

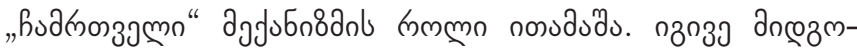

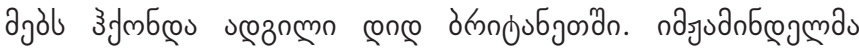

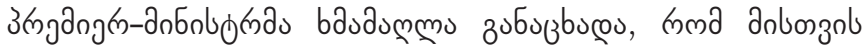
зо о

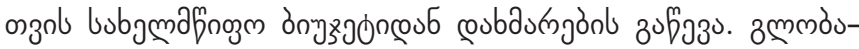

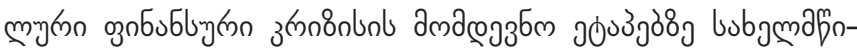

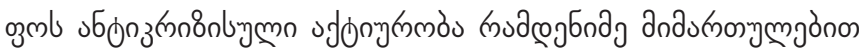

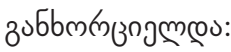

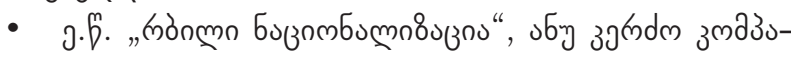

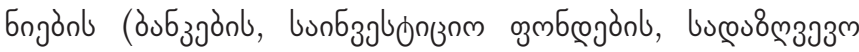

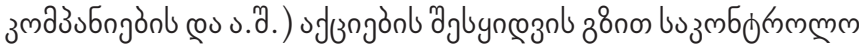

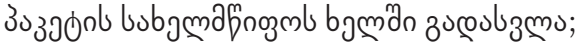

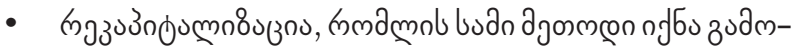

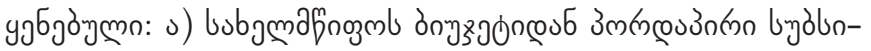

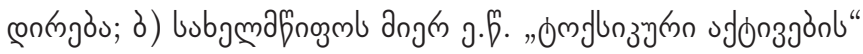

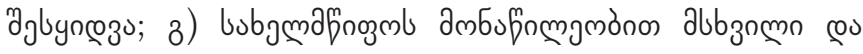

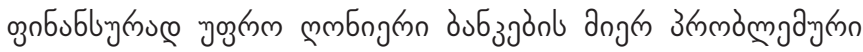

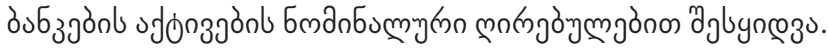

8̈дmuмб

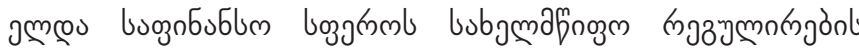

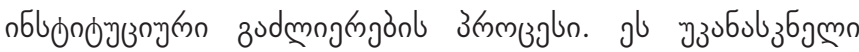

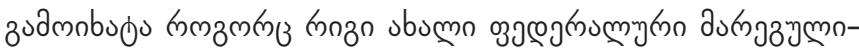

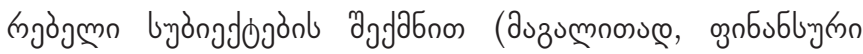
amabubygrgonl anfmegònb buogegm (Financial Oversight

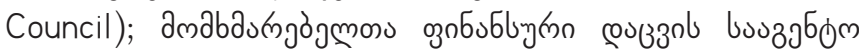
(Consumer Financial Protection Agency, CFPA), gr্⿻コ一

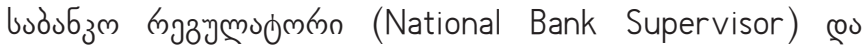

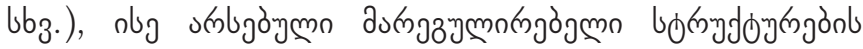

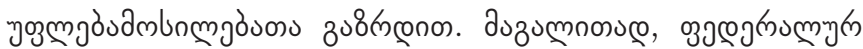

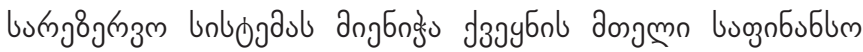

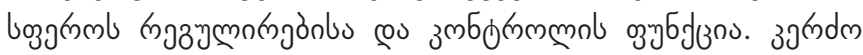

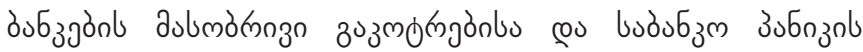

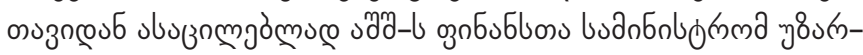

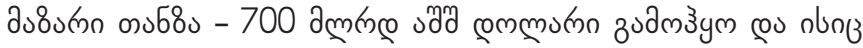

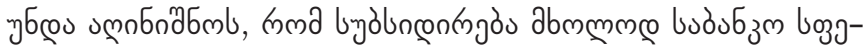

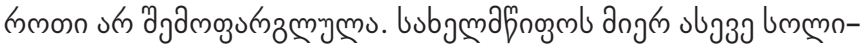

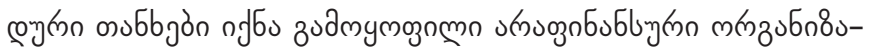

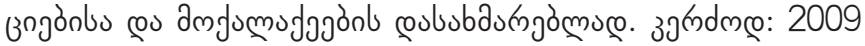

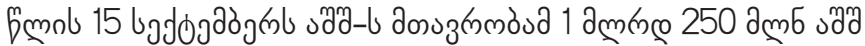

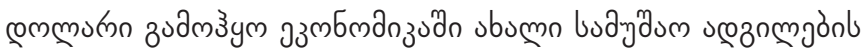

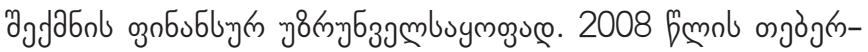

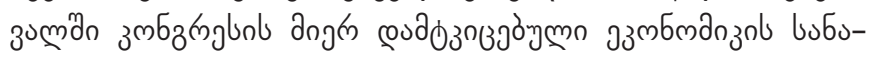

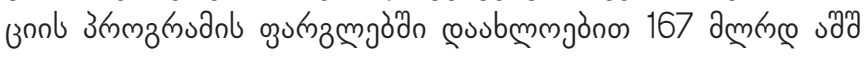

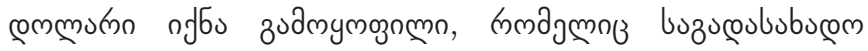

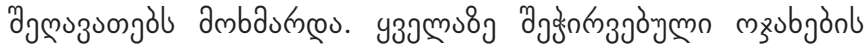

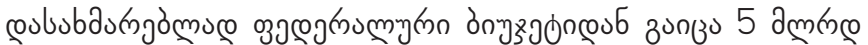

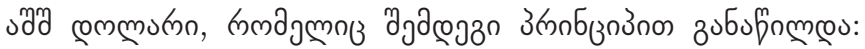

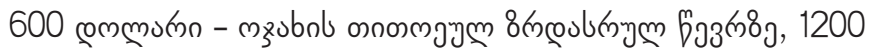

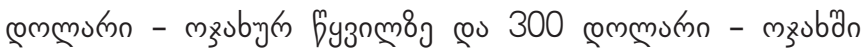

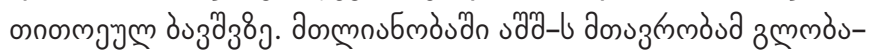

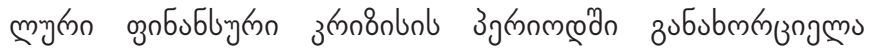

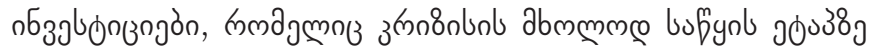

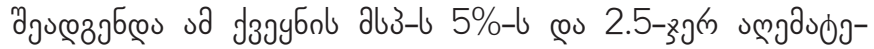

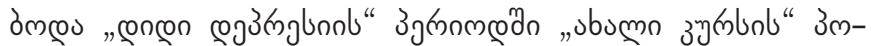

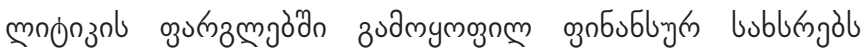

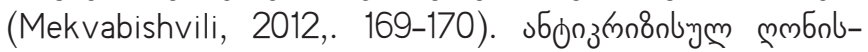

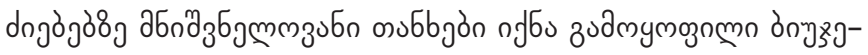

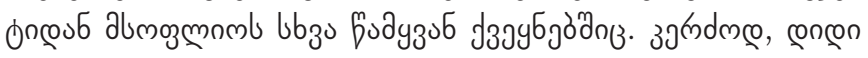

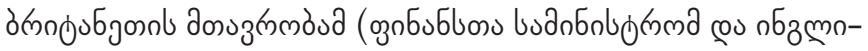

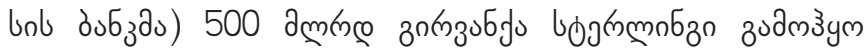
bu

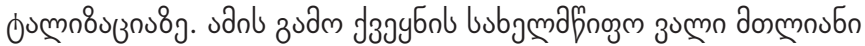

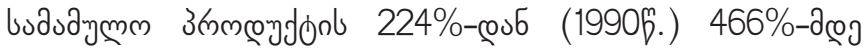
zun8umgou (2009\%.) (www.bank-of-england.co.uk/publications/news/2009/019.html).

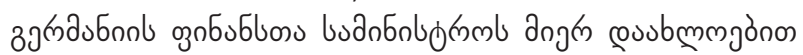

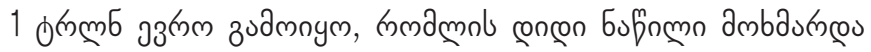

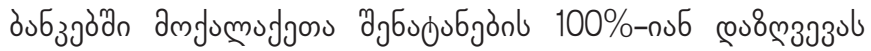

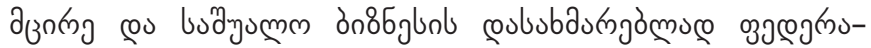

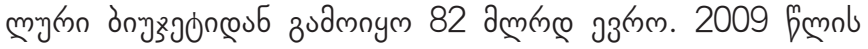

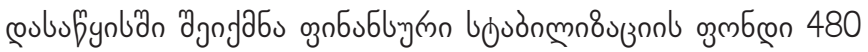

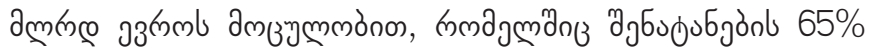

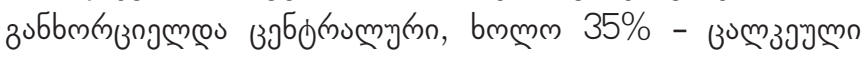

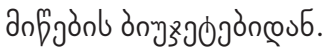

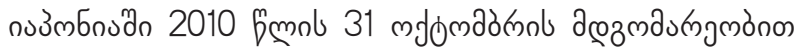

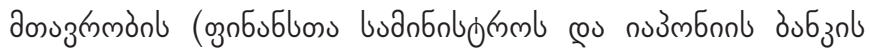

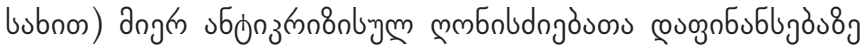

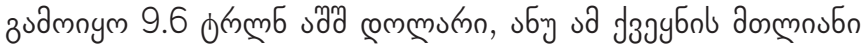

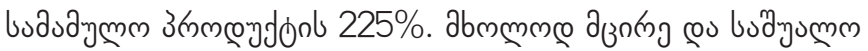

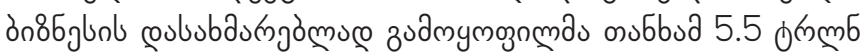

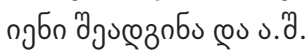

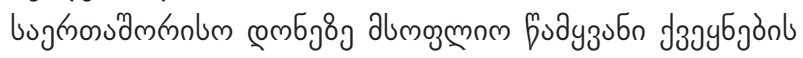

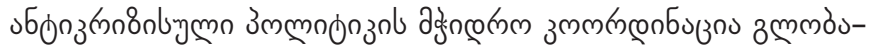

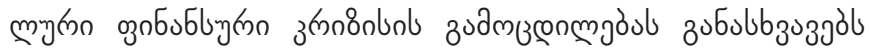

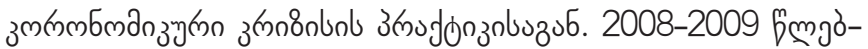

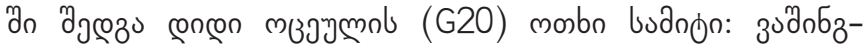

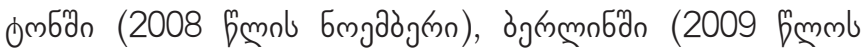




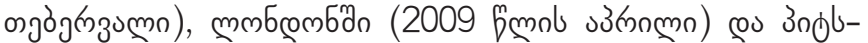

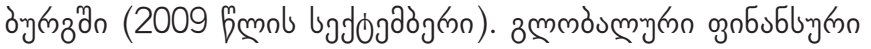

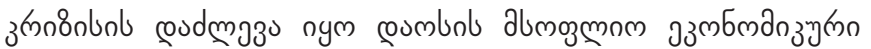

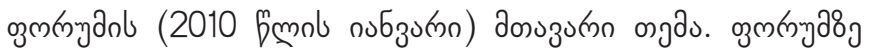

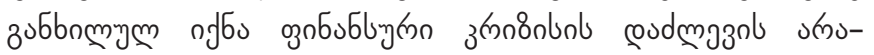

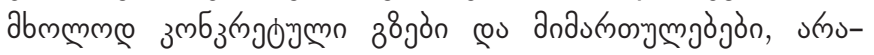

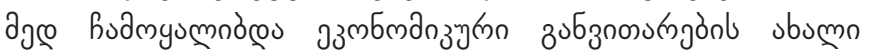

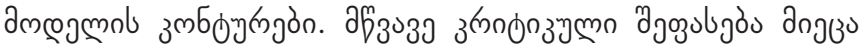

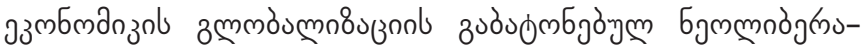

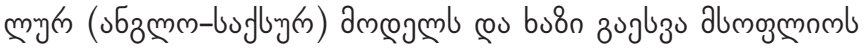

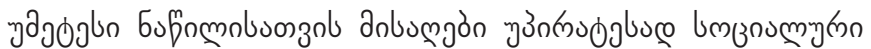

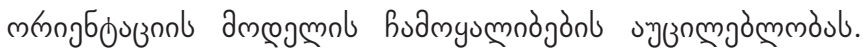

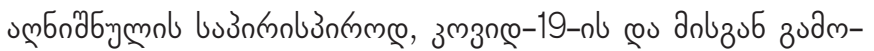

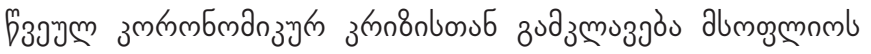

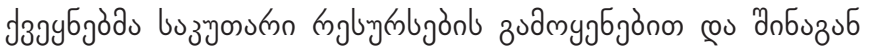

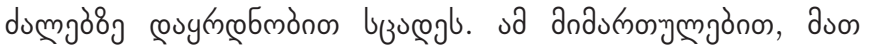

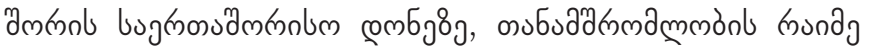

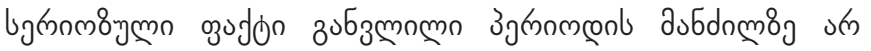
euognflunträ̀ymu.

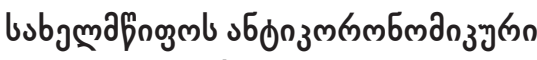 gmbnldngàjòn}

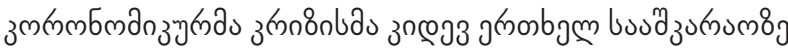

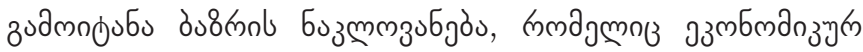

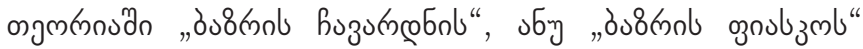

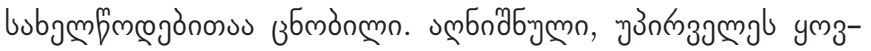

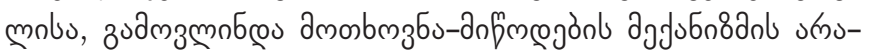

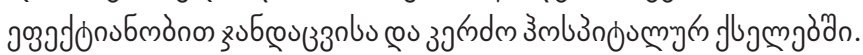

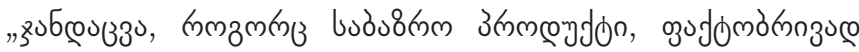

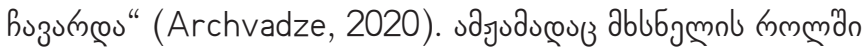

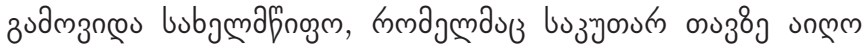

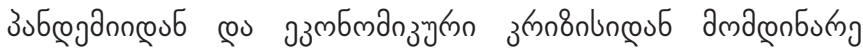

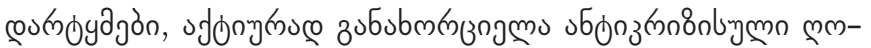

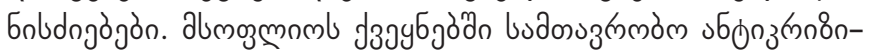

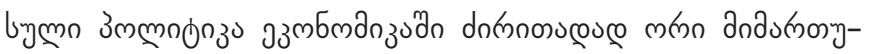

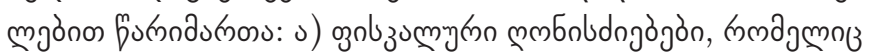

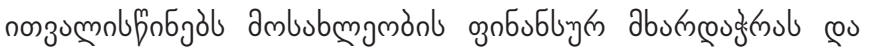

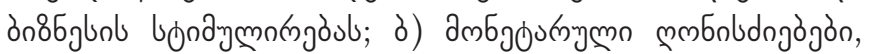

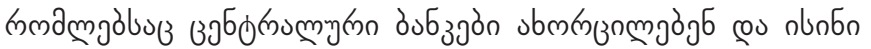

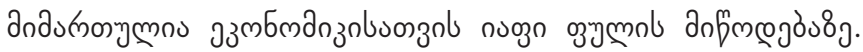

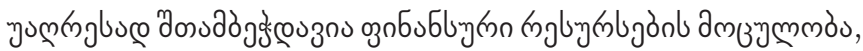

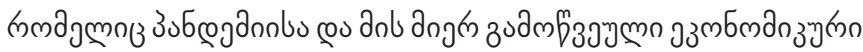

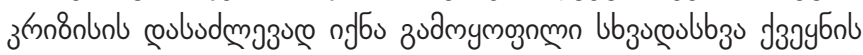

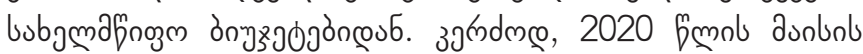

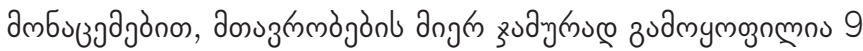

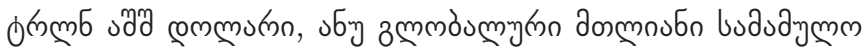

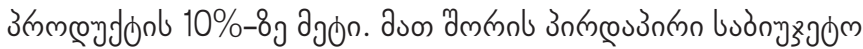

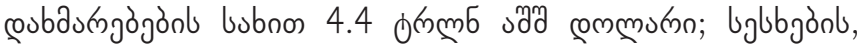

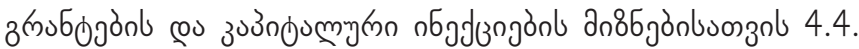

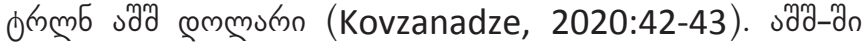

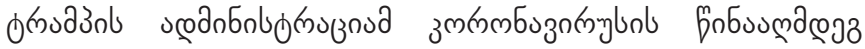

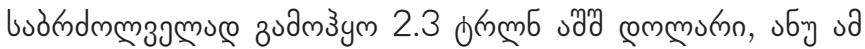

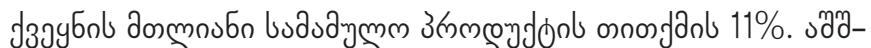

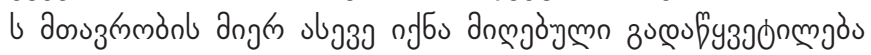

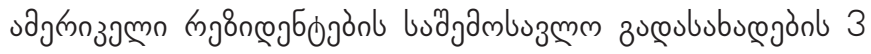

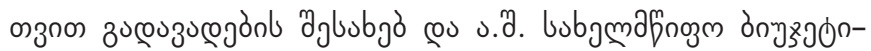

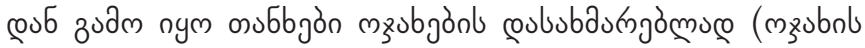

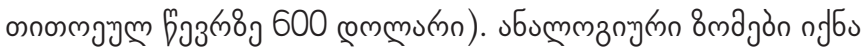

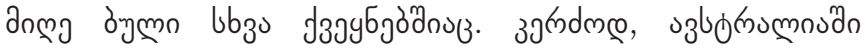

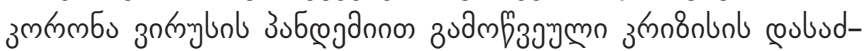

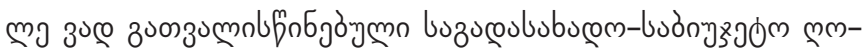

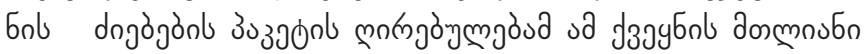

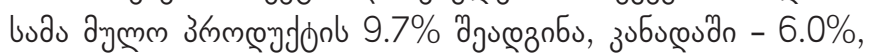

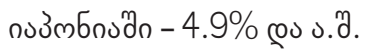

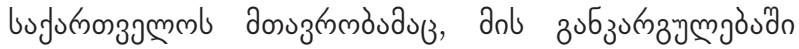
s

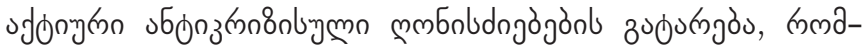

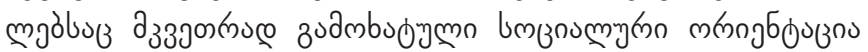

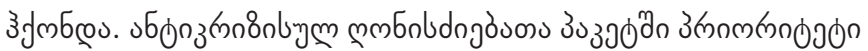

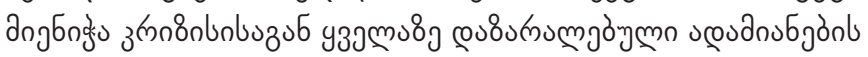

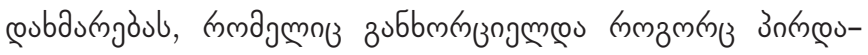

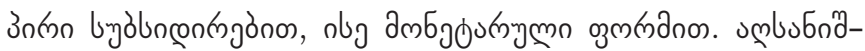

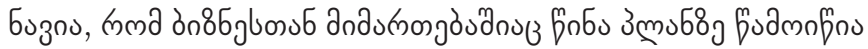

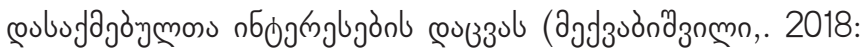
12-13).

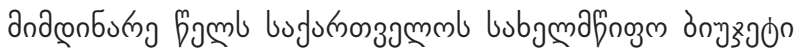

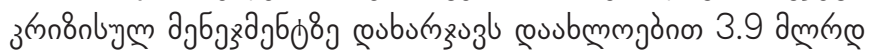
mumbl. duon गेmnol:

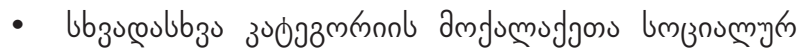

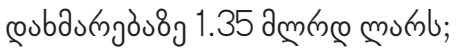

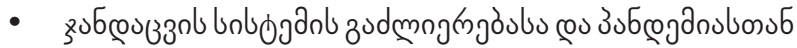
jஙndmmu8̊ - 350 amb mumb;

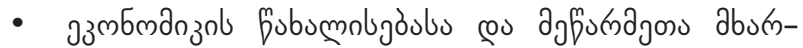

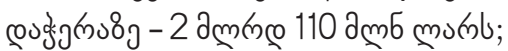

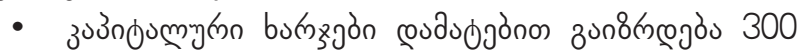

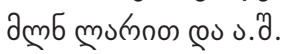

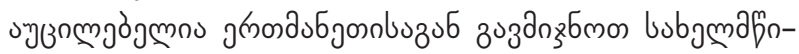
gुmb sбonz

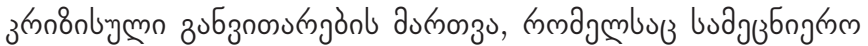

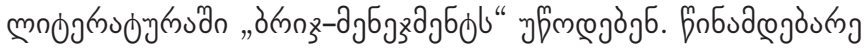

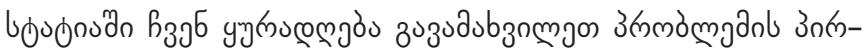

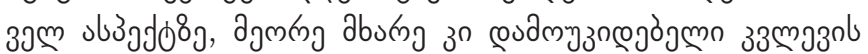
buzubn agndmgàs aubegl.

\section{cos336s}

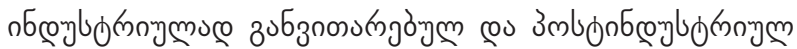

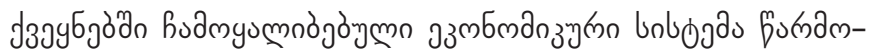

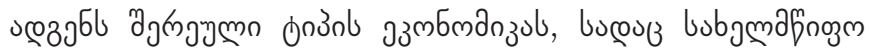




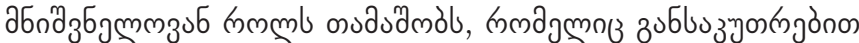

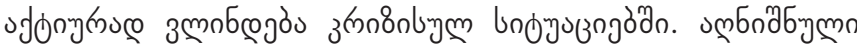

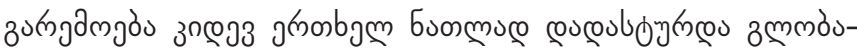

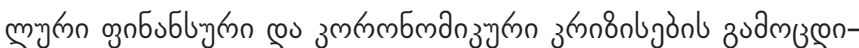

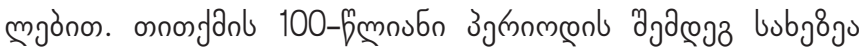

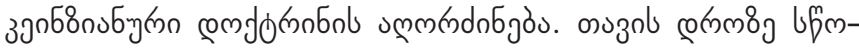

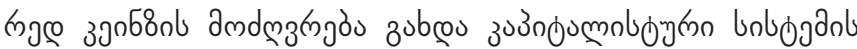

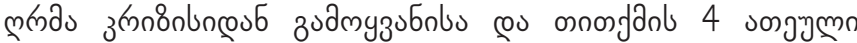

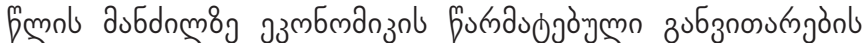

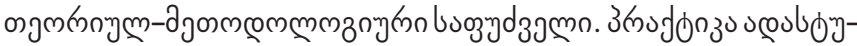

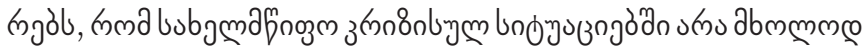

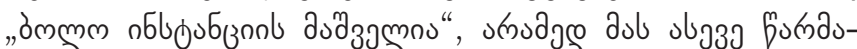

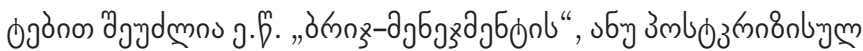

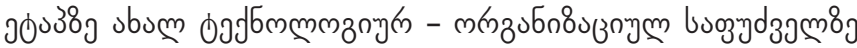

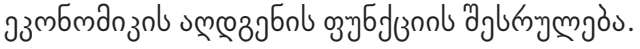

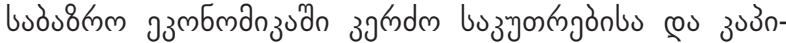

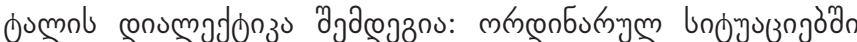

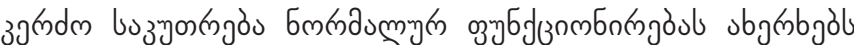

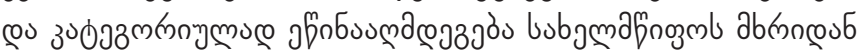

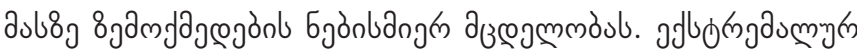

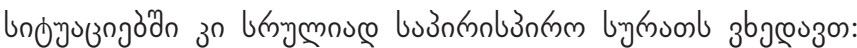

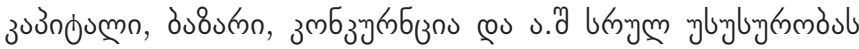

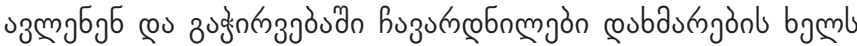

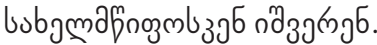

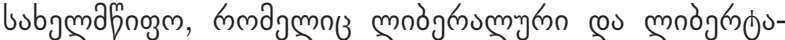

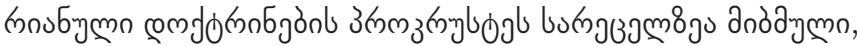

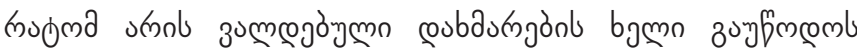
उ

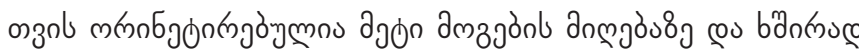

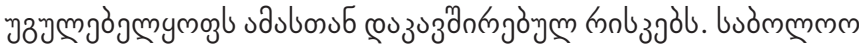

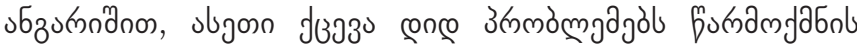

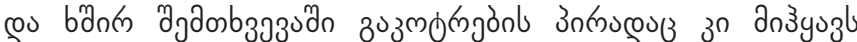

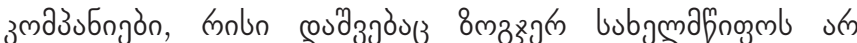

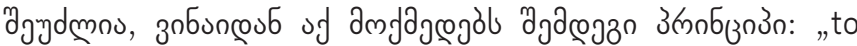

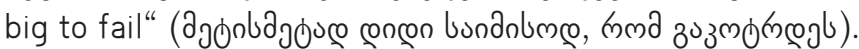

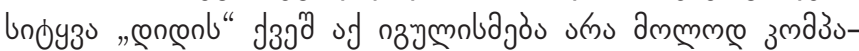

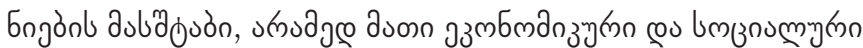

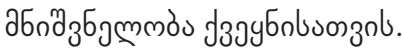

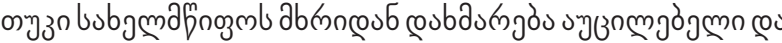

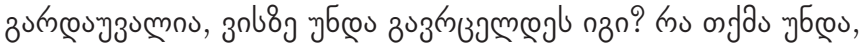

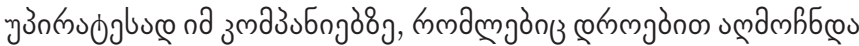

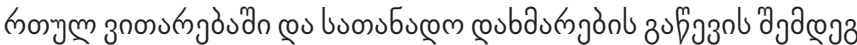

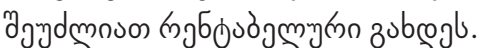

абпда

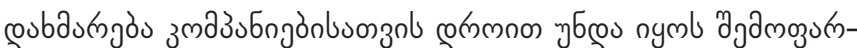

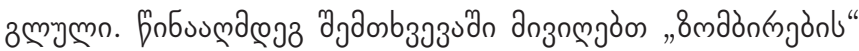

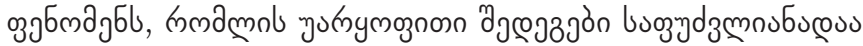

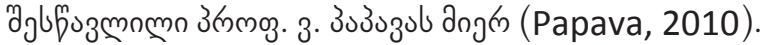

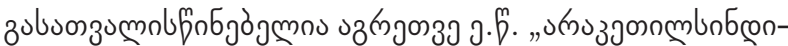

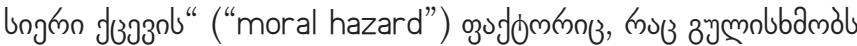

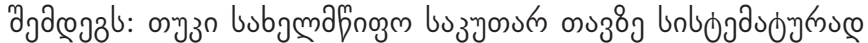

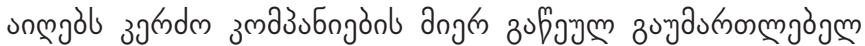

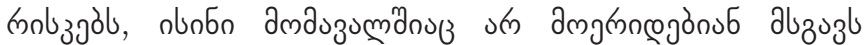
jagejóall.

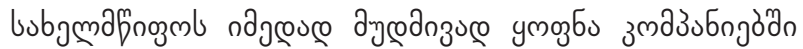

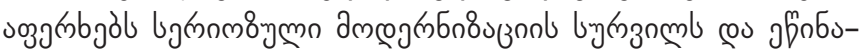
ง мам

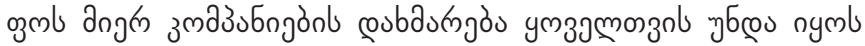

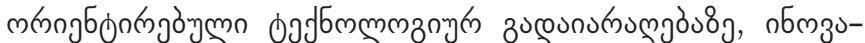

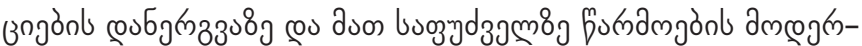

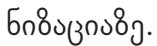

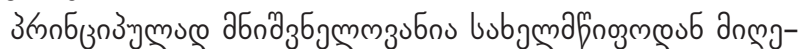

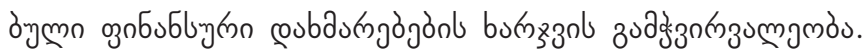

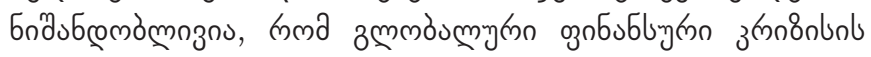

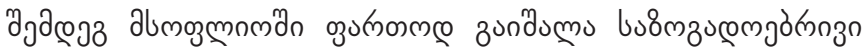

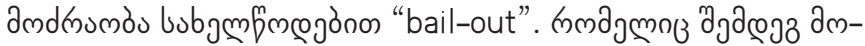

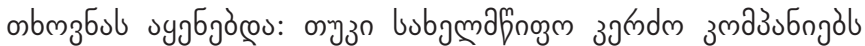

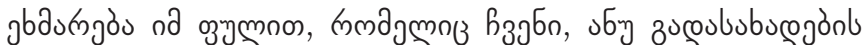

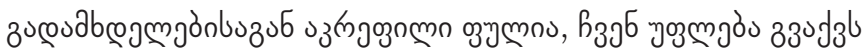

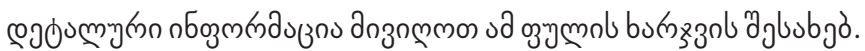

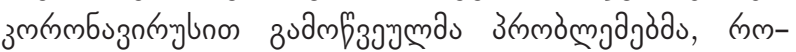

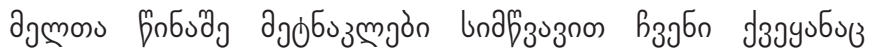

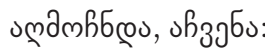

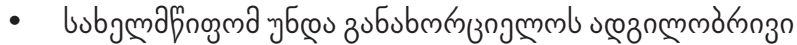

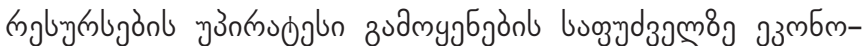

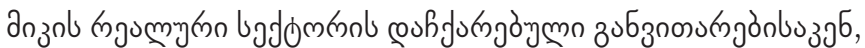

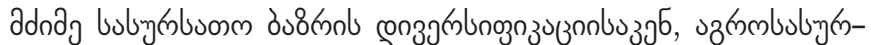

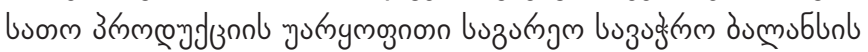

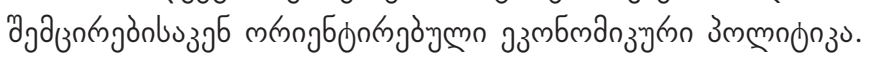

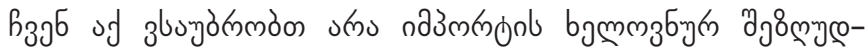

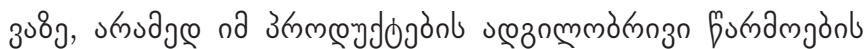

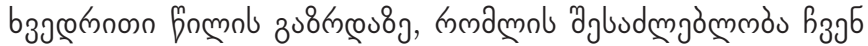

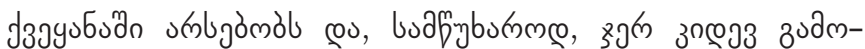

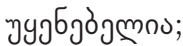

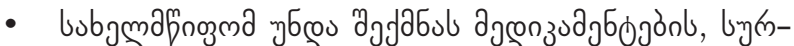

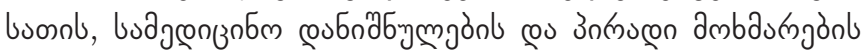
bо пrod

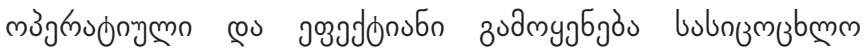

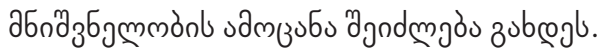

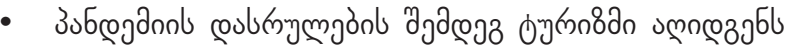

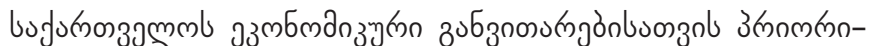

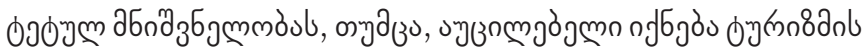

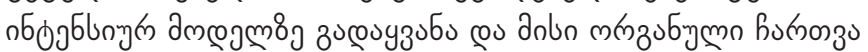

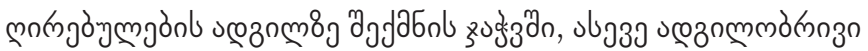

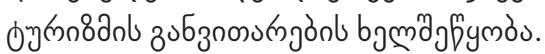

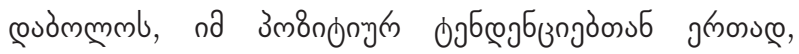

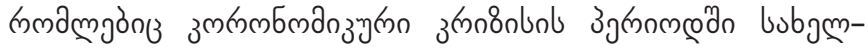

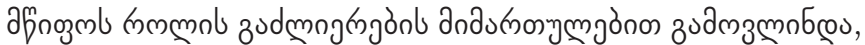

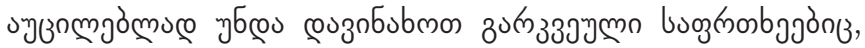

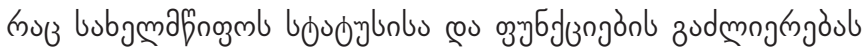




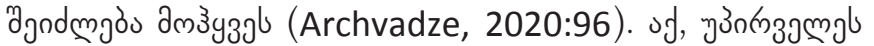

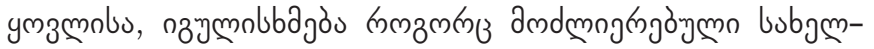

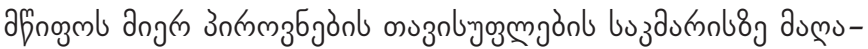

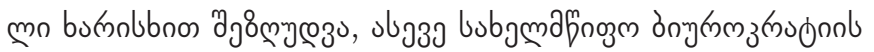
angங mòn

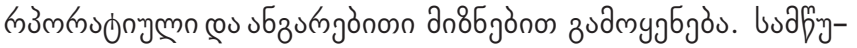

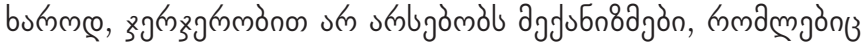

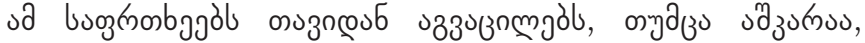

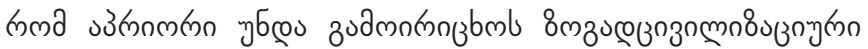

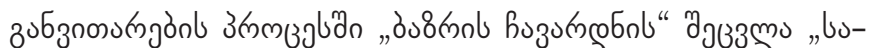

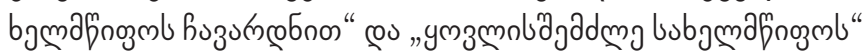

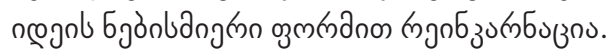

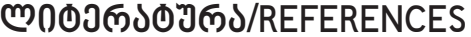

Archvadze, I., (2020). Transformation of state function in the postpandemic period. Economy and Business, N2; (In Georgian) Kovzanadze, I., (2020). Economic Challenges and Prospects in the Background of COVID-19 Infection. Economycs and Business, N2; (In Georgian)

Mekvabishvili, I., (2018). Financial Crises of the Globalization Era and the Georgian Economy. Tbilisi, Intellect; (In Georgian) Mekvabishvili, E., (2020). The global coronopmic crisis and its impact on the Georgian economy. Economycs and Business, N2; (In Georgian)

Papava, V., (2010). Zombie-ing of the Necroeconomy (The Impact of the Global Financial and Economic Crisis on the Countries of the Black Sea Region). Tbilisi (In Georgian).

Monitoring the World Crisis (2010). (In Russian)

https://for.ge/view/182671/koronomika-da-ekonomika-krizisis-winase.html (https://bit.ly/3676pyd/

https://bit.ly/2TcSmoqu

https://bit.ly/2ABme7A

www.bank-of-england.co.uk/publications/news/2009/019.html 